\title{
Zum Zirkel von Beschreibung und Normierung, dargestellt am Beispiel der deutschen Interpunktionslehre
}

\section{On the Circle of Description and Prescription - Based on the Example of German Theory of Punctuation}

Karsten Rinas

\begin{abstract}
This article offers a sketch of the development of German theory of punctuation. It is shown that within this process of codification of orthographic rules there is an interaction of descriptive and prescriptive approaches which can be regarded as an analogy of the hermeneutic circle. In regard to this, there is no principal difference between the standardization of orthography and the development of grammars. Further, it is shown that the theory of punctuation should be regarded as a part of the Grammar of German written language.
\end{abstract}

\section{Keywords}

orthography, punctuation, descriptive and prescriptive linguistics, linguistics of written language 


\title{
1 Einleitung
}

In diesem Beitrag soll gezeigt werden, dass sich selbst im Bereich der angeblich ,willkürlichen' Orthographie eine Wechselwirkung deskriptiver und normativer Ansätze beobachten lässt, eine Art Zirkel zwischen Empirie und Normierung, der sich als Analogon zum hermeneutischen Zirkel deuten ließe. ${ }^{1}$ Daraus folgt, dass zwischen Orthographie und Grammatikschreibung kein prinzipieller Unterschied besteht. Dies soll hier am Beispiel der Entwicklung der Interpunktionslehre illustriert werden. Des Weiteren wird dafür argumentiert, diese Lehre als einen Teilbereich der Grammatik der deutschen Schriftsprache zu betrachten.

Diese Einschätzungen stehen in einem gewissen Widerspruch zu noch heute häufiger vertretenen Ansichten über die Schriftsprache und die Grammatikschreibung, die hier zunächst skizziert werden sollen.

\subsection{Eine Ansicht über die Schriftsprache}

Die Frage, ob die Schriftsprache ein wichtiger oder bloß ein peripherer Gegenstand der Sprachwissenschaft sei, wird kontrovers diskutiert. Nach wie vor zeigt sich hierbei der Einfluss Ferdinand de Saussures, der die gesprochene Sprache als den eigentlichen, zentralen Gegenstand der Linguistik definierte (1967[1916]: 28-30), ${ }^{2}$ was zur Folge hatte, dass die Schrift „allenfalls als Stiefkind“ angesehen wurde, „dem im Bereich der Linguistik als wissenschaftlicher Disziplin keine Aufmerksamkeit geschenkt zu werden brauchte“ (Coulmas 1981: 12). Auch heute wird diese Reserviertheit gegenüber der ,künstlichen' Schrift mitunter zum Ausdruck gebracht, beispielsweise von Meinunger (2008: 161):

\begin{abstract}
„Die Kommunikation mit Hilfe von Schrift ist ein immenser kultureller Fortschritt, gleichsam eine gesellschaftliche Revolution. Sie ist eine geniale bewusste Erfindung des Menschen. Aber es gab und gibt auch Menschen ohne Schrift und folglich ohne Rechtschreibung. [...] Die Sprache gehört nach manchen Definitionen dagegen sogar zwingend zum Menschsein. Sie ist quasi eine biologische Erfindung, ein Produkt der Natur, zumindest entstanden in einem gemeinsamen untrennbar miteinander verbundenen Evolutionsprozess. Die Schrift hat der Mensch dagegen bewusst entwickelt."
\end{abstract}

\subsection{Eine Ansicht über die Grammatikschreibung}

Eine weit verbreitete Auffassung der modernen Linguistik besagt, dass die Sprachwissenschaft rein deskriptiv sein solle und folglich nicht normativ/präskriptiv sein

1 Zu dem Zirkel-Konzept der Hermeneutik vgl. etwa Grondin (2016).

2 Diese kritische Einstellung der Schriftsprache gegenüber wurde auch schon von Hermann Paul propagiert, des Weiteren etwa auch von Leonard Bloomfield; vgl. Coulmas (1981: 21-24). 
dürfe (vgl. etwa Hudson 1984: 15-19). Entsprechend wird dann auch die Grammatikschreibung als ein rein deskriptives Unterfangen konzipiert, eine Auffassung, die in jüngerer Zeit etwa Maitz \& Elspaß (2007: 517) artikuliert haben:

„Am Anfang war nicht der Duden, an dem sich die deutsche Sprachgemeinschaft zu orientieren hatte, sondern die deutsche Sprachgemeinschaft, deren Sprachgebrauch der Duden u.a. möglichst vollständig zu erfassen versuchten. Daraus folgt dann aber auch, dass es im Grunde nicht nur überflüssig, sondern geradezu sinnlos ist, deutschen Muttersprachlerinnen - außer natürlich (zukünftigen) Deutschlehrerinnen, die in ihrem Beruf ohne explizite Regelbeschreibungen nicht auskommen können - deutsche Grammatiken in die Hand zu geben bzw. ihnen auf Grund dieser Grammatiken Ratschläge zu geben, welche Regeln sie beim Sprechen oder Schreiben beachten sollten.“

\subsection{Gegenthesen}

Den Auffassungen, die exemplarisch durch die Zitate von Meinunger sowie Maitz \& Elspaß belegt wurden, ${ }^{3}$ sollen hier folgende Thesen entgegengestellt werden:

i) Auch die Schrift und die Schriftregularitäten entwickeln sich evolutiv.

ii) Die Beschreibung der Schriftregularitäten ist ein Teil der Grammatikschreibung, in dem sich deskriptive und normative Aspekte ebenso durchdringen wie in allen anderen Bereichen (etwa in der Formenlehre).

These i) impliziert, dass auch Schriftregularitäten keineswegs grundsätzlich ,künstlich konstruierte' oder, willkürliche“ Erscheinungen sind und dass sie folglich mit der gleichen Berechtigung untersucht werden können wie andere Bereiche der Sprache.

These ii) impliziert unter anderem, dass die weit verbreitete Forderung einer scharfen Abgrenzung der deskriptiven Sprachwissenschaft von normativen Ansätzen nicht haltbar ist.

Beide Thesen sind keineswegs vollkommen neu, sondern werden seit dem ausgehenden 20. Jahrhundert (wieder) häufiger vertreten. ${ }^{4}$ These i) wird in ausführlicher Form im Rahmen Schriftlinguistik propagiert, zu deren Begründung u.a. das bereits zitierte Werk von Coulmas (1981) einen Beitrag geleistet hat (vgl. ferner etwa Dürscheid 2012). Und in Bezug auf orthographische Regeln hat etwa schon Mentrup (1983: 45) bemerkt, dass sie „präskriptive Regeln“ seien, „die auf deskriptiven Regeln beruhen." Auch These ii) wird spätestens seit den 1990er Jahren zumindest sporadisch artikuliert; vgl. etwa Helbig (1996), Bergenholtz (2001), Klein (2004), Rinas (2011: Kap. 7 u. 12).

3 Sowohl die Ansicht von Meinunger als auch die von Maitz \& Elspaß wurden im Kontext der Debatte um das sprachkritische Werk Bastian Sicks artikuliert. Zu einer kritischen Einschätzung dieser Debatte - vor allem auch der Beiträge von linguistischer Seite - vgl. Rinas (2011).

4 In gewisser Hinsicht handelt es sich hierbei um eine Rückkehr von Auffassungen, die bereits der älteren Grammatikschreibung zugrunde lagen. Insbesondere hat es dort keine scharfe Trennung von Deskription und Präskription gegeben. Vgl. hierzu etwa Henne (1984). Vgl. auch Rinas (2011: Kap. 3 u. 5). 
Im Folgenden soll zunächst die Entwicklung der Interpunktionslehre skizziert werden, da sich hier diverse Momente beobachten lassen, welche die Thesen i) und ii) stützen.

\section{Skizze der Geschichte der deutschen Interpunktionslehre}

\subsection{Die rhetorische Periodenlehre}

Die ältesten Beiträge zur deutschen Interpunktionslehre entstammen dem 15. Jahrhundert. Hierzu gehören die Darstellungen von Niklas von Wyle (um 1462), Heinrich Steinhöwel (1473) und Friedrich Riederer (1493). ${ }^{5}$ Diese Beiträge sind stark der rhetorischen Periodenlehre verpflichtet, welche bereits in der antiken Rhetorik entwickelt wurde (vgl. etwa Quintilianus, Buch IX, Kap. 4, §§122-125). Dieser Lehre zufolge ist eine ,Periode“ eine selbständige Wortverbindung. Diese kann untergliedert werden in ,Cola“ und ,Commata'. Ein ,Colon“ ist hierbei definiert als eine Wortgruppe, die zwar rhythmisch vollständig ist, jedoch nicht als vollständig empfunden wird (oder wie es oft heißt: ,keine eigene Bedeutung hat ${ }^{\text {- }}$ was natürlich cum grano salis zu nehmen ist). Und ein ,Comma' wird aufgefasst als eine Wortverbindung, die zwar durch Sprechpausen abgegliedert ist, die jedoch nicht die rhythmische Vollständigkeit eines Colons aufweist. Ein Beispiel:

(1) Er wollte nicht kommen, obwohl er, laut Maria, Zeit gehabt hätte.

Das gesamte Beispiel (1) bildet eine selbständige Wortverbindung und kann somit als Periode aufgefasst werden. Der konjunktional eingeleitete Nebensatz ${ }^{6}$ (obwohl er, laut Maria, Zeit gehabt hätte) ist rhythmisch (relativ) vollständig bzw. eigenständig, wird jedoch als inhaltlich nicht eigenständig empfunden; er kann somit als Colon bestimmt werden. Die parenthetische Angabe (laut Maria) lässt sich zwar intonatorisch bzw. durch Sprechpausen absondern, ist aber weder rhythmisch noch inhaltlich eigenständig und kann somit als Comma bestimmt werden.

Die antike Periodenlehre arbeitet also vorwiegend mit folgenden Kriterien:

(2) rhetorische Kriterien der Periodenlehre:

Selbständigkeit-Unselbständigkeit / Abgeschlossenheit-Unabgeschlossenheit (un)vollständiger Sinn

Rhythmus / Sprechpause

Diese Kriterien sind allerdings vage und schwer anwendbar, weshalb etwa auch die

5 Vgl. hierzu Höchli (1981).

6 Diese Explikation ist allerdings anachronistisch, denn es ist gerade charakteristisch für die rhetorische Periodenlehre, dass sie nicht mit modernen syntaktischen Kategorien wie Haupt- und Nebensatz oder Satzgliedern operiert; nicht einmal die Wortartenlehre spielt in dieser traditionellen Konzeption eine Rolle. 
auf das Beispiel (1) bezogenen Klassifikationen anfechtbar sind. Aber diese tentative Konzeption bildet nun einmal den Ausgangspunkt der Interpunktionslehre, sodass sie aus fachhistorischer Sicht berücksichtigt werden muss.

Wie aus diesen Explikationen hervorgeht, bezeichneten ,Periode', ,Colon“ und ,Comma' ursprünglich Wortgruppen. Schon in der Spätantike kam es jedoch zu einer terminologischen Verschiebung bzw. Erweiterung: Mit diesen Termini wurden fortan (auch) die Zeichen benannt, die dazu dienten, diese Wortgruppen abzugrenzen. $^{7}$

Aus fachhistorischer Sicht ist nun insbesondere die Frage von Interesse, wann die Periodenlehre durch präzisere Konzeptionen ersetzt oder ergänzt wurde, wann also andere Kriterien als die in (2) aufgelisteten in Gebrauch kamen. Insbesondere kommen hierbei die folgenden moderneren syntaktischen Kriterien in Betracht:

(3) grammatische/syntaktische Kriterien (im strengeren Sinne):

Rekurrieren auf, interpunktionssensitive“ Wortarten (v.a. Konjunktionen);

Satzgliedkonzeption (einzelne Satzglieder: Subjekt, Objekt, Adverbialbestimmung usw.);

grammatisch-logische Satzdefinition $($ Satz $=$ Subjekt + Prädikat $)$;

Hauptsatz - Nebensatz; Nebenordnung - Unterordnung u.a.

Kurzum: Wann und in welcher Weise erfolgte der Übergang von (2) zu (3)?

In einem anderen Beitrag (Rinas 2012) habe ich ausgeführt, dass alle deutschen Interpunktionslehren des 15. und 16. Jahrhunderts der rhetorischen Periodenlehre folgen. Erst im Laufe des 17. Jahrhunderts kommt es zu ersten Ergänzungen und Modifikationen. Ein frühes Beispiel ist die Lehre von Brücker (1620), wo immerhin bereits eine syntaktisch-semantische Zusatzkonzeption bemüht wird, indem das (relativ neue) Zeichen Semikolon mit Adversativitätskontexten korreliert wird; vgl. Rinas (2015). Dies ist allerdings nur ein erster zaghafter Ansatz. Ansonsten bleibt Brücker - wie auch alle seine Zeitgenossen - vollständig der rhetorischen Periodenlehre verhaftet.

\subsection{Syntaktische Fundierung im ausgehenden 17. Jahrhundert}

Zu bedeutenden konzeptionellen Weiterentwicklungen der deutschen Interpunktionslehre kommt es erst im ausgehenden 17. Jahrhundert (vgl. Rinas 2014). Hierbei spielen zunächst weder Grammatiken noch Rechtschreiblehren, sondern vor allem Briefrhetoriken eine wichtige Rolle, ${ }^{8}$ insbesondere die Interpunktionslehre in Christian Weises Werk Curiöse Gedancken Von Deutschen Brieffen... (1691: 238-271). Weise gliedert hier die

7 Vgl. etwa Bieling (1880: 10), Parkes (1993: 21-23).

8 Dies illustriert, dass gerade in der älteren Geschichte der deutschen Interpunktionslehre der Einfluss des rhetorischen Schrifttums berücksichtigt werden muss - ein Umstand, den die bisherige Forschung nicht ausreichend reflektiert hat; vgl. hierzu Rinas (2012). 
Periode - durchaus traditionell - in den (spannungsaufbauenden),Vor-Satz' (protasis) und den (spannungslösenden) ,Nach-Satz' (apodosis), wobei er allerdings betont und herausarbeitet, dass diese jeweils konjunktional eingeleitet seien, sodass komplexe, aus Vor- und Nachsatz bestehende, Perioden bei ihm typischerweise durch zweigliedrige Konjunktionen verbunden sind. Diese beiden Teile der komplexen Periode sollen nun nach Weise durch ein Colon (:) getrennt werden, wie im folgenden Beispiel:

(4) Ob zwar meine Gelegenheit nicht zulassen will/ denselben mit kostbaren Tractamenten zu begegnen: dennoch habe ich/vor meine Persohn/nichts unterlassen wollen/wodurch mein auffrichtiges Gemüthe/möglichster massen/kan erwiesen werden. (S. 247)

Somit kommt Weise das Verdienst zu, eine konsequente und systematische Berücksichtigung der Konjunktionen als interpunktionsrelevante Wortart in die deutsche Interpunktionslehre eingeführt zu haben.

Darüber hinaus fordert Weise, dass wenn ein Vor- oder Nachsatz seinerseits wieder in Vor- und Nachsatz zerfällt, diese Teile ,zweiter Stufe' nicht durch ein Colon, sondern durch ein Semicolon abzutrennen seien, wie in dem folgenden Beispiel:

(5) Vor-Satz: Nachdem ich die Ehre genossen/zu seiner Hochzeitlichen Festivität/ als ein lieber Gast/invitiret zu werden:

Nach-Satz: Als habe nicht allein ich/vor meine Persohn/solches über die massen hoch aestimiret; sondern ich kan gleichfalls nicht beschreiben/ was die gelibten Meinigen vor eine Vergnügung daraus geschöpffet haben." (S. 249)

Anders - und etwas anachronistisch - formuliert: Weise fordert, Nebensätze ersten Grades mit anderen Zeichen abzugrenzen als Nebensätze zweiten Grades. Hier manifestiert sich ein Gespür für hierarchische Strukturen, das unser heutiges System an Differenziertheit deutlich übertrifft.

Weises klare, konsequent hierarchiebezogene Konzeption wurde intensiv rezipiert (vgl. Rinas 2014). Zunächst wurde sie jedoch ausschließlich in Briefrhetoriken übernommen, doch gelangte sie - vermutlich durch die Vermittlung von Freyer (1722) - bald auch in Rechtschreiblehren und Grammatiken. Auch in vielen späteren Interpunktionslehren - etwa den Beiträgen von Adelung und Duden - hat Weises Konzeption ihre Spuren hinterlassen. Einige Versatzstücke hielten sich mit erstaunlicher Zähigkeit, insbesondere die Kolonregel für Perioden (vgl. Abschnitt 4.1).

\subsection{Weiterentwicklungen im 18. und 19. Jahrhundert}

Im Laufe des 17. und 18. Jahrhunderts wurde die Weisesche Lehre modifiziert und erweitert. Dies bedeutet allerdings nicht, dass sie hierdurch verbessert worden wäre. 
Das Gegenteil war der Fall: Die klare hierarchische Konzeption Weises wurde durch die Hereinnahme zusätzlicher Kriterien - etwa semantische Kriterien, Satzlänge, Sprechpausen - immer unübersichtlicher, sodass oft nicht klar war, wann welches Kriterium greifen soll. Sehr deutlich zeigt sich diese Verworrenheit in Adelungs Konzeption (etwa in Adelung 1782: 791-798; vgl. Rinas 2014). Dennoch bildete sich gerade im Anschluss an Adelung eine Art Standardlehre heraus, die in der ersten Hälfte des 19. Jahrhunderts massenhaft referiert wurde (vgl. Rinas 2016). Ihr Kern lässt sich folgendermaßen zusammenfassen:

- Punkt, Kolon, Semikolon, Komma sind hierarchisch gestuft nach ihrer Stärke bzw. Pausenlänge.

- Der Punkt markiert das Ende eines Satzes und/oder einer Periode.

- Das Kolon trennt den Vorsatz vom Nachsatz; letzterer ist i.d.R. mit so eingeleitet.

+ Kriterium der Satzlänge: Das Kolon steht in langen Perioden; wenn jedoch Vor-/Nachsatz kurz sind, genügt das Komma.

+ Ankündigungsfunktion für direkte Rede, Beispiele u.ä.

- Das Semikolon gliedert vor allem komplexe Vorsätze oder Nachsätze.

+ diverse semantische Konstellationen, etwa Kausalität oder Adversativität (Hier gab es große Variation!)

- Komma = ,schwächstes Zeichen', trennt ,kleinere Glieder; typische Kontexte: Relativsätze, Einschübe, Koordinationen, Appositionen, bestimmte Konjunktionen (daß, weil, wenn u.a.).

Bis auf einige Detailfragen (etwa Koordinationen, Infinitivkonstruktionen...) $)^{9}$ war dieses System bereits recht konsolidiert. Es bildete auch die Grundlage für den Entwurf einer Interpunktionslehre von Duden (1876), welcher eine wichtige Grundlage für die Standardisierung der Interpunktion bildete.

Wir können den historischen Abriss an dieser Stelle abbrechen. Im Folgenden soll auf die Frage eingegangen werden, inwiefern bei der Entwicklung der deutschen Interpunktionslehre normative und deskriptive Momente interagierten. Dies soll zunächst anhand von Stellungnahmen von Interpunktionstheoretikern und anschließend an zwei Fallbeispielen diskutiert werden.

\section{Stellungnahmen von Interpunktionstheoretikern}

Bereits in Christian Weises Interpunktionslehre findet sich eine interessante Bemerkung (1691: 271):

9 Gerade bei diesen Detailfragen setzt im 19. Jahrhundert allerdings eine intensive Diskussion ein, wobei diverse syntaktische ,Subtilitäten' teils auf hohem theoretischen Niveau analysiert werden; vgl. hierzu Rinas (2016). 
„Ich schreibe zwar nichts neues: doch hat iemand diese Regeln vor dieser Zeit eben auff mein Fundament gesetzet/so kan ich wol sagen/daß ich ihn nicht gesehen/vielweniger ausgeschrieben habe."

Hier hebt Weise also die Eigenständigkeit seiner Leistung hervor, und diese Versicherung kann man ihm - auch im Hinblick auf die Einschätzung durch Zeitgenossen - durchaus glauben (vgl. Rinas 2014). In welchem Sinne kann Weise dann aber behaupten, dass er ,nichts Neues' schreibe? Dies ist offenbar dahingehend zu verstehen, dass Weise den Anspruch erhebt, die bereits vorhandene Lehre und/oder den herrschenden Usus präziser erfasst zu haben.

Deutlichere Stellungnahmen, die in diese Richtung weisen, finden sich in größerer Zahl im 19. Jahrhundert. So schreibt etwa Herling (1827: 406) zu seinen Interpunktionsregeln:

„Bei der Aufstellung obiger Regeln ist der herrschende Gebrauch vorzugsweise berücksichtigt. Freilich ist dieser noch oft schwankend, vornehmlich in dem Gebrauche des Puncts, der durch die Grammatiken nur mit einer kurzen Regel abgefertigt wird."

Hier manifestiert sich also der Anspruch, den herrschenden Gebrauch zu berücksichtigen, diesen aber zugleich in Zweifelsfällen durch die Regeln zu konsolidieren.

Ausführlicher sind die konzeptionellen Darlegungen von Weiske (1838), wo sehr entschieden eine Anbindung an die sich gerade etablierende satztheoretisch fundierte Syntax gefordert wird:

„Wenn aber selbst in der neusten Zeit der Gebrauch der Interpunction noch ein unsicherer ist und über die Bedeutung und den Werth der einzelnen Zeichen noch eine große Meinungsverschiedenheit herrscht, so liegt der Grund davon in dem Mangel einer richtigen Satztheorie, welche zugleich als Norm für den Gebrauch der Interpunction dienen muß." (S. 4)

„Begegnen wir aber bei Betrachtung einer jeden Sprache der merkwürdigen Erscheinung, daß, obwohl wir als Grund dazu nur ein dunkles Gefühl annehmen müssen, doch überall der Geist ein gewisses systematisches Gebäude aufgeführt hat, das der philosophirende Verstand nur bewundern kann; so dürfen wir auch wohl bei der Interpunction voraussetzen, daß eine innere, wenn auch unbewußte, Nothwendigkeit ihren Gebrauch geregelt habe, und daß auch bei ihr eine wissenschaftliche Betrachtung ein nicht unfruchtbares Feld finden wird. Und in der That hat es sich gefunden und wird die weitere Ausführung zeigen, daß selbst bei nicht ganz deutlichen Begriffen über das Wesen und den Zweck der Interpunction dieselbe ein eigenthümliches System in sich entwickelt hat, welches auf der Grundlage der Satzbildung beruhend mit derselben in der innigsten Harmonie steht. Wir werden deshalb nicht nur jeder Theorie derselben die Regeln der Satzbildung zu Grund legen müssen, sondern auch ohne Schwierigkeit die Interpunction selbst von diesem Standpuncte aus betrachten können, ohne von dem Hergebrachten sehr abzuweichen." (S. 5) 
Hier wird für die Interpunktionslehre geradezu eine Forschungsperspektive eröffnet, indem betont wird, dass die Interpunktion keineswegs willkürlich konzipiert sei, sondern einer ,innere[n], wenn auch unbewußte[n], Nothwendigkeit“ folge und somit ein „eigenthümliches System“ bilde, welches adäquat unter Anwendung der neueren Satztheorie rekonstruiert werden könne. Und weil dieses natürlich gewachsene System mit der Satzbildung „in der innigsten Harmonie“ stehe, sei es möglich, es theoretisch zu rekonstruieren, „ohne von dem Hergebrachten sehr abzuweichen“. - Die in Abschnitt 1.3 formulierten Thesen werden also bereits von Weiske sehr deutlich herausgearbeitet.

Ähnlich geartete Ausführungen finden sich auch bei Becker (1839). Becker bemerkt zwar zunächst, dass sich die Orthographie nur „als ein zur Darstellung der gesprochenen Rede erfundenes Mittel“ darstelle und daher „weit weniger anziehend“ sei „als die eigentliche Grammatik“ (iii). In seinen nachfolgenden Ausführungen eröffnet er jedoch eine neue Perspektive: Die Orthographie „ist nach ihrer Entstehung und nach ihrem ganzen Wesen nichts Anderes, als ein treues Gegenbild der darzustellenden Rede; und darum werden in ihr alle Besonderheiten der gesprochenen Sprache ausgeprägt“ (v). Dies gelte auch für die Interpunktion: Deren Zeichen seien „Zeichen für die Tonverhältnisse der Sätze“ (vii), welche wiederum „der organische Ausdruck ihrer logischen Form" seien (viii):

\begin{abstract}
„Diese Betrachtung der Thonverhältnisse gibt uns nun über die logische Form der zusammengesetzten Sätze, und [...] über die logischen Verhältnisse derselben überhaupt Aufschlüsse, zu denen man auf andern Wegen nie gelangen würde. Indem wir diese Betrachtung nur in Beziehung auf die Interpunktion anstellen, ergibt sich aus ihr weit mehr, als wir eigentlich suchten." (viii)
\end{abstract}

Somit sei es Becker selbst klar geworden, dass „die Beziehungen, in denen die Orthographie zu der eigentlichen Grammatik steht, weit inniger sind, als man gewöhnlich glaubt“ (ix). Man könne hier „eine von ihm [Becker] selbst früher kaum geahndete lebendige Entwickelung“ finden (ix).

Auch hier wird also ein Forschungsprogramm formuliert: Aufgabe der Interpunktion ist es, die „logische Form“ der Sätze widerzuspiegeln. Dem nachzugehen, ist gerade auch aus syntaktischer Sicht ergiebig; es werden natürliche Korrelationen von ,logischer Form' und Interpunktionskonventionen herausgearbeitet.

Ein besonders interessantes Beispiel einer empirischen Herangehensweise bietet die Studie ,Drei Kommaregeln statt vieler“ von Wittmütz (1850). Das Hauptziel dieser Studie besteht darin, das Regelwerk für den Kommagebrauch zu reduzieren, was in den Vorschlag von nur drei Regeln mündet. Dieses Regelwerk entwickelt Wittmütz auf der Grundlage einer Korpusauswertung, wobei ausgewählte Texte von „unsern großen Dichtern und Prosaikern“ (S. 18) herangezogen wurden. ${ }^{10}$ Der Anspruch ist

10 Wittmütz reflektiert hierbei, dass die Ausgaben dieser Texte nicht unbedingt die Original-Interpunktion der Autoren wiedergeben müssen (S. 13f., 29). 
hierbei, die vorgefundenen Gebrauchsweisen auch und gerade in ihren Variationen zu berücksichtigen. In diesem Sinne fordert Wittmütz nachdrücklich, den Gebrauchsweisen der ,Klassiker' mit Respekt zu begegnen (S. 18f.) und sie nicht auf der Folie der oft rigorosen Schulgrammatiken abzuwerten: „Die Lehrer nehmen sonst gern an, was von den gepriesenen Meistern der Kunst herkommt; in der Kommafrage scheinen sie indess ihre pädagogischen Bedenken bis weiter noch nicht aufgeben zu wollen" (S. 61).

Auf eine eingehendere Diskussion von Wittmütz' Regeln wollen wir verzichten. Hier sei nur erwähnt, dass Wittmütz bemüht ist, auch stilistische Variation zu berücksichtigen, sodass er in Varianten unterschiedliche Sichtweisen oder ,Absichten des Schreibenden' sieht.

Aber nicht alle Theoretiker standen dem Usus in der deutschen Interpunktion so positiv gegenüber. Eine kritischere Einschätzung formulierte Götzinger (1839):

\begin{abstract}
„Offenbar entbehrt die deutsche Zeichensetzung der Leichtigkeit und hat zufolge ihres wissenschaftlichen Anstriches etwas höchst Unpraktisches. Ohne bestimmte Kenntnis des Satzbaues, die doch erst erworben seyn will, kann kein Deutscher die Zeichen richtig setzen, während der Franzose nur dem Ohre zu folgen braucht, und dann nicht irren wird.“ (S. 417f.)
\end{abstract}

Bemerkenswert ist allerdings, dass sich Götzinger trotz dieser Einschätzung nicht dazu entschließt, eine eigene Interpunktionslehre - etwa nach französischem Vorbild - zu entwerfen, sondern vielmehr einen Abriss folgen lässt (S. 416-432), der sich an die in Abschnitt 2.3 skizzierte, zu Götzingers Zeit bereits allgemein etablierte, Standardlehre hält. Götzingers Einwand ist somit eher akademischer Natur. Götzinger ist Realist genug, um den Kern der weit verbreiteten Standardlehre anzuerkennen.

Innerhalb dieser Standardlehre gab es freilich noch Variationen, was insbesondere von Schulmännern beklagt wurde. So schreiben Hergang et al. (1852: 352):

„Sogar bedeutende Grammatiker weichen in der Aufstellung der Regeln über den Gebrauch des Comma, des Colon und Semicolon von einander ab. [...] Für die Schule sollte man sich endlich nach bestimmten Reglen umsehen! - Es wäre dies nur dadurch zu erreichen, daß sich die Vorsteher der Schulconferenzen eines größern Landstriches, nach vorausgegangener Berathung mit den Conferenzgliedern, über eine festzusetzende Orthographie vereinigten.“

Andererseits reflektieren auch Hergang et al. den weit entwickelten Usus, wenn sie die Bedingungen formulieren (ebd.), unter denen eine Festsetzung der Orthographie erfolgen müsste: „durchgängig das Gebräuchlichste festhalten“; „vor Neuerungen müßte man sich auf's sorgfältiste hüten“; „nur da, wo sich das Neuere bereits vielfach Geltung verschafft hat, sind ihm auch die Pforten der Schule nicht zu verschließen“.

Um die Mitte des 19. Jahrhunderts wird diese konservative, am Usus orientierte Haltung von allen führenden Theoretikern geteilt. ${ }^{11}$ Versuche radikaler Umgestal-

11 Es gab allerdings seit der Mitte des 19. Jahrhunderts radikalere Vertreter einer - von Jacob Grimm 
tungen werden allenfalls noch von einigen Revolutionären und Schwärmern unternommen, wie etwa von dem Journalisten und Revolutionsagitator Friedrich Wilhelm Held $^{12}$ (1844: 110-128), der generell eine ,Revolution der Orthographie‘ fordert und auch für die Interpunktionszeichen Regeln formuliert, die stark vom Usus abweichen:

- Komma $=$ reines Pausenzeichen - wird nur nach Gefühl gesetzt! (S. 117f.)

- Semikolon: trennt wirkliche Sätze (mit Subjekt + Prädikat) in Perioden (S. 113)

Es ist sogar eine Verdoppelung erlaubt, um Grade zu verdeutlichen:

Der Man ;;welcher gestern zu mir kam; und den ich schon for zwei Jaren gesehen hate;; brachte mir einen sonderbaren brif.

Dies war jedoch um die Mitte des 19. Jahrhunderts nicht mehr als eine intellektuelle Spielerei, die keine Aussicht auf Erfolg hatte.

Wir können die hier exemplarisch wiedergegebenen Auffassungen folgendermaBen zusammenfassen:

- Spätestens im 19. Jahrhundert stellen die meisten Interpunktionstheoretiker den geltenden Usus nicht mehr in Frage, sondern erheben vielmehr den Anspruch, ihn in ihren theoretischen Entwürfen zu reflektieren.

- Sie fordern ,lediglich' eine empirisch adäquate Erfassung sowie eine bessere theoretische Durchdringung dieses Usus.

- In Zweifelsfällen wird zumeist eine Vereindeutigung angestrebt, um den Usus zu homogenisieren.

Diese Tendenzen wirkten sich auch in zwei Fallbeispielen aus, die im Folgenden diskutiert werden sollen.

\section{Zwei Fallbeispiele}

\subsection{Der neue Prosastil und der Niedergang der Periode}

Bereits in Abschnitt 2.2 wurde die von Christian Weise (1691) eingeführte Kolonregel für die Periode angeführt, der zufolge in einer komplexen Periode Vorsatz und Nachsatz durch ein Kolon (:) zu trennen seien. Das Kolon wurde dadurch zum Periodenzeichen par excellence, und gerade diese Regel sollte sich mit besonderer Zähigkeit halten. Man findet sie auch noch in Konrad Dudens erstem Entwurf einer

\footnotetext{
inspirierten - historisch-etymologisch orientierten Orthographie, zu denen etwa Karl Gustav Andresen und Karl Weinhold gehörten. Allerdings konzentrierten sich diese Theoretiker auf die historisch begründete Wortschreibung, während sie im Bereich der Interpunktion kaum anderes zu bieten hatten als den wenig hilfreichen Appell, sparsamer zu interpungieren. Daher konnten sie in diesem Bereich auch keine nachhaltige Wirkung entfalten, und selbst im Bereich der Wortschreibung blieb ihr Einfluss begrenzt. Vgl. hierzu auch Rinas (i.D.).

12 Über das Wirken von Held vgl. Haacke (1969).
} 
Interpunktionslehre (1876: 199). Und selbst in der 17. Auflage des Rechtschreibdudens (1973: 32, R 72) heißt es noch: „Der Doppelpunkt steht vor dem Nachsatz im durchgegliederten mehrfach zusammengesetzten Satz (Periode).“ - Diese Regel war damals gewiss schon hoffnungslos veraltet, und es ist bezeichnend, dass als Beispiel hierfür ein Beleg von einem Autor des 19. Jahrhunderts herhalten musste (ebd.):

„Wo dir Gottes Sonne zuerst schien; wo dir die Sterne des Himmels zuerst leuchteten; wo seine Blitze dir zuerst seine Allmacht offenbarten und seine Sturmwinde dir mit heiligem Schrecken durch die Seele brausten: da ist deine Liebe, da ist dein Vaterland (E. M. Arndt).“

Erst in der 18. Auflage des Rechtschreibdudens von 1980 findet sich diese Kolonregel nicht mehr, sodass man der Weiseschen Regel - zumindest in der Theorie - eine erstaunliche Langlebigkeit attestieren kann.

Dieser bemerkenswerte Konservativismus der Mainstream-Lehre sollte allerdings nicht darüber hinwegtäuschen, dass es bereits in der ersten Hälfte des 19. Jahrhunderts Theoretiker gab, die die Abschaffung dieser Regel forderten. So postuliert bereits Lorberg (1823: 51f.), das Kolon ausschließlich in der uns heute vertrauten Weise, nämlich als Ankündigungszeichen, zu gebrauchen, und er bemerkt: ${ }^{13}$

„Auf die Einwendung, es sey ohne ein solches Hülfszeichen nicht gut möglich, lange Perioden richtig zu lesen, möchte man wohl mit Recht erwiedern können, es sey dem Geiste der Deutschen Sprache und den Forderungen eines guten Stils weit angemessener, wenigstens in Prosa nicht so unmäßig lange Perioden zu bilden. Überhaupt dürfte es schwer seyn, für alle die verkünstelten und verschrobenen Wortfügungen, deren man freilich selbst aus den neuern und neuesten Schriften eine zahllose Menge zusammenbringen könnte, eine genügende Interpunctions-Theorie zu erfinden. Halten wir uns vielmehr an das Einfache!“

Lorberg fordert hier somit eine Anpassung der Interpunktionslehre an die sprachlichen Gegebenheiten seiner Zeit. Da das Schreiben hochkomplexer, ,verschrobener Perioden nicht mehr üblich sei, bestehe auch für das Kolon als Zeichen für komplexe Perioden kein Bedarf mehr. Lorberg will somit in der Interpunktionslehre einen Sprachtrend - die Bildung einfacherer, übersichtlicherer Sätze - reflektieren und diesen Trend damit zugleich befördern.

Ist Lorbergs Vorgehensweise nun deskriptiv oder normativ? Diese Frage lässt sich nicht eindeutig beantworten, denn Lorbergs Argumentation umfasst beide Aspekte: Reflexion des Sprachusus sowie Festigung und Beförderung eines gewünschten Sprachzustands. Die simple dichotomische Gliederung in deskriptive und normative Ansätze greift hier offenkundig zu kurz.

13 Sehr ähnlich argumentiert auch Herling (1827: 406). 


\title{
4.2 Ausbreitung und Reflexion des Gedankenstrichs
}

Auch an einem älteren Beispiel aus dem 18. Jahrhundert lässt sich das Wechselspiel von Deskription und Präskription gut studieren, und zwar anhand der allmählichen Ausbreitung des Gedankenstrichs. Dieser Vorgang ist aus kulturhistorischer Sicht eines der interessantesten Phänomene der Interpunktionsgeschichte, dem allerdings bislang in der Forschung nur wenig Beachtung geschenkt wurde.

Die Ausbreitung dieses Zeichens ist gewiss nicht den Interpunktionslehren zu verdanken. Sie war vielmehr assoziiert mit dem Aufkommen neuer ästhetischer Auffassungen und Schreibweisen, die sich im Kontext der literarischen Strömungen des Rokoko, der Anakreontik, des Pietismus und der Empfindsamkeit ausbildeten und in der deutschen Literatur schließlich im ,Sturm und Drang“ kulminierten. Hierbei kam es zu ,sprunghaften' Schreibweisen, in denen der Gedankenstrich und funktional ähnliche typographische Mittel wie [=] oder [...] eine wichtige Funktion ausübten. Als recht früher Beleg hierfür kann ein Gedicht des anakreontischen Dichters Johann Georg Peter Möller dienen:

„Siehe! hie heb ich den Arm itzt langsam vor dir in die Höhe,

Freundinn, und schwöre dir ewige Treu, $===$

Da seh ich sie $==$ welch ein Glück? = = sie die längsterwünschte Zähre,

Die dir von schamfreyen Wangen herrollt.“ (Möller 1752: 131f.)

Diese Schreibweisen wurden schon früh kritisiert. So heißt es in einer spöttischen Abhandlung „über den neuen ,Witz“ im Kulturleben“ (Anonymus 1754: 157f.):

\begin{abstract}
„Die neuesten witzigen Köpfe pfeiffen ein etwas von Mädgen und Wein, um zu zeigen, daß sie wissen, es sey ein Anacreon auf der Welt gewesen, und daß sie den Namen eines Hagedorns und Gleims gehört haben [...] So haben unsere neuesten witzigen Köpfe lauter windschiefe Gedanken, davon keiner auf den andern passet [...] Wo ihnen die Gedanken fehlen, wo sie auch nicht Worte hinsetzen können, da lassen sie den Raum mit Strichen erfüllen. Das ist gewiß ganz was neues, und hierin ist der Witz auf der höchste getrieben. Soll ich meinen Lesern ein Muster davon vorlegen? doch nein, ich möchte sonst - - - - -

Denn man kan nicht alles sagen, es fehlen oft die Ge- - - -
\end{abstract}

danken.“

Dies war allerdings eine literarische Kritik. Die Interpunktionslehren begannen diese Erscheinung erst später zu reflektieren. Ein frühes Beispiel findet sich in der Sprachlehre von Bodmer (1768: 126), wobei in den knappen Ausführungen auch typographische Varianten berücksichtigt werden: „Dem Leser mehr zu denken zu geben, als man sagt, es sey, daß man gerne abbrechen will, oder daß man sich fürchtet, die Sache herauszusagen, setzet man einen oder zween kurze Querstriche; oder man setzet für die Strichgen nur etliche Pünktchen nach einander.“ Dieser Beschreibung fügt Bodmer eine kritische Anmerkung hinzu: „Verfasser, die Mangel 
an Gedanken haben, oder sie nicht auszudrücken wissen, behelfen sich öfters mit dieser Nothhülfe."

Ähnliche - mit einer stilkritischen Warnung versehene - Ausführungen finden sich etwa auch bei Heynatz (1770: 69f.), Felbiger (1774: 23f.) und Rossberg (1774: 160-162), wobei sich diese Autoren zugleich um detailliertere und differenziertere Gebrauchsbeschreibungen für den Gedankenstrich bemühten. Auch am Ende des 18. Jahrhunderts waren Warnungen vor einer allzu intensiven oder missbräuchlichen Anwendung dieses Zeichens weiterhin üblich; vgl. etwa Adelung (1788: 388-392) und Moritz (1796: 28f.).

Auch bei diesem Phänomen zeigt sich also eine Durchdringung von Deskription und Präskription: Die Interpunktionstheoretiker reagieren - etwas verzögert - auf eine neue Entwicklung, die sich vor allem in der neueren Literatur ihrer Zeit manifestiert. Sie versuchen dieses Phänomen analytisch zu durchdringen, indem sie die gängigen Gebrauchsweisen eingehender beschreiben. Zugleich warnen sie aber auch vor einem extensiven und achtlosen Gebrauch des Gedankenstrichs, um den ,kultivierten' Umgang mit diesem Zeichen zu fördern.

\section{Neuere Beiträge zur Interpunktionstheorie}

Auch in der modernen Schriftlinguistik ist eine Abgrenzung normativer und deskriptiver Ansätze verbreitet. Sie findet sich üblicherweise in der terminologischen Unterscheidung von (deskriptiver), Graphematik' und (normativer) ,Orthographie (vgl. etwa Dürscheid 2012: 126f.). Solange diese Differenzierung als ein grober Orientierungsrahmen angesehen wird, ist hiergegen nichts einzuwenden, doch birgt sie die Gefahr in sich, als strikte dichotomische Gliederung interpretiert zu werden. Eine scharfe Abgrenzung normativer und deskriptiver Ansätze wird jedoch den Gegebenheiten nicht gerecht, denn auch in modernen schriftlinguistischen Beiträgen sind normative und deskriptive Aspekte - notwendigerweise - miteinander verbunden. Dies sei hier an zwei Beispielen illustriert.

Primus (1997) versucht die komplexen Regeln für die Kommasetzung im Deutschen zu vereinfachen, indem sie das Regelwerk auf folgende Prinzipien reduziert:

„Ein Komma zwischen einem einfachen oder komplexen Ausdruck A und einem einfachen oder komplexen Ausdruck B ist regulär genau dann, wenn (a) und (b) oder (a) und (c) gelten:

a) Es gibt einen Satzknoten, der A und B dominiert.

b) Zwischen A und B interveniert eine syntaktische oder semantische Satzgrenze.

c) A und B sind koordiniert, und die Koordination ist nicht durch eine echte koordinierende Konjunktion gekennzeichnet." (S. 478f.)

Auf eine eingehende Diskussion dieser Konzeption soll hier verzichtet werden. Wir begnügen uns mit dem Hinweis auf ein von Primus selbst diskutiertes Problem: Eine grundlegende Kommaregel nach dem Rechtschreibduden (1991: Regel 109) besagt, 
dass das Komma bei koordinierten vollständigen Hauptsätzen gesetzt wird, auch wenn diese durch eine Konjunktion verbunden sind, vgl. etwa:

(6) Die Kerzen wurden angezündet, und man machte es sich bequem.

Diese Regel ergibt sich nicht zwingend aus den von Primus formulierten Prinzipien, ist also durch diese nicht motiviert. Entsprechend vertritt Primus die These, dass es sich bei diesem Kommagebrauch um „eine reine Konvention“ handle. Zudem sei nach Primus sein Ausnahmestatus auch durch Fehlerstatistiken gestützt (S. 482). ${ }^{14}$

$\mathrm{Ob}$ dies wirklich ausreichende und überzeugende Gründe sind, die Kommasetzung bei koordinierten vollständigen Hauptsätzen als ,reine Konvention' zu bestimmen, sei dahingestellt. Grundsätzlich ist jedoch Primus' Argumentation vollkommen legitim: Primus bemüht sich um eine möglichst einfache Rekonstruktion der Prinzipien des Kommagebrauchs im Deutschen, und als sie feststellt, dass diese Prinzipien mit der orthographischen Norm nicht völlig übereinstimmen, erklärt sie die von ihrem System nicht erfassten Regeln für problematisch, wobei sie sich auf Fehlerstatistiken stützt, um zu illustrieren, dass auch die Sprecherintentionen mit den geltenden orthographischen Regeln nicht kongruieren. Man könnte auch sagen, dass ihre theoriegeleitete Untersuchung sie zur Aufdeckung eines ,Zweifelsfalls‘ führt, was sie dazu veranlasst, eine entsprechende Modifizierung der geltenden Norm in Betracht zu ziehen.

Das Beispiel zeigt, wie eine empirisch-theoretische Untersuchung zu Resultaten führen kann, die eine Modifikation der geltenden Norm nahe legen oder forcieren können. Insofern gibt es in Primus' Beitrag einen direkten Übergang von einem deskriptiven Ansatz zu tendenziell normativen Erwägungen. Dies ist keine Schwäche dieses Beitrags. Dieses Oszillieren zwischen Deskription und Norm ist vielmehr nicht nur berechtigt, sondern letztlich sogar unvermeidlich.

Als weiteres Beispiel sei hier die umfassende linguistische Rekonstruktion des Interpunktionsgebrauchs von Bredel (2011) angeführt. Bredel knüpft - implizit - an die schon im 19. Jahrhundert vertretene Position an, dass die Interpunktion einer ,inneren Logik' folge, dass ihr also ein System zugrunde liege, welches es zu rekonstruieren gelte (vgl. Abschnitt 3). Diesen Anspruch verknüpft Bredel mit neueren Auffassungen der strukturalistischen Linguistik, und zwar so konsequent, dass sich ihre einleitend formulierte Zielsetzung fast schon wie ein Manifest des Strukturalismus liest: Das Ziel sei, die „Interpunktion als System“ zu rekonstruieren, wobei gelten solle:

„Interpunktionszeichen, also die Elemente des Systems, bilden eine von anderen Schriftzeichen abgegrenzte Klasse von Zeichen, die in definierter, nicht in willkürlicher Beziehung zueinander stehen. Das heißt: Jedes Einzelelement ist eindeutig, erfüllt also innerhalb des Systems

14 Diese kritische Sicht auf die Kommasetzung in diesem Kontext hat sich auch im Zuge der Rechtschreibreform durchgesetzt, denn nach den neuen Regeln ist die Kommasetzung zwischen selbständigen durch und verbundenen Hauptsätzen fakultativ; vgl. etwa Deutsche Rechtschreibung (2006: 82). 
eine spezifische Funktion [...]. Die Funktion von Einzelzeichen ist außerdem konsistent: Ein Zeichen ist in derselben Umgebung nicht einmal da, einmal nicht da [...]. Darüber hinaus ist die Funktion jedes Einzelelements diskret: Dieselbe Funktion kann nicht von unterschiedlichen Elementen übernommen werden; zwischen verschiedenen Markierungen von Einschüben (Komma, Klammer, Gedankenstrich) muss es also ebenso wie zwischen Sätzen in Texten und in freistehenden Zeilen einen angebbaren Unterschied geben."

„Systeme bilden Strukturen: Strukturen beschreiben die Eigenschaften von und die Beziehungen zwischen Einzelelementen, die ein System zusammenhalten. Änderungen eines Einzelelements wirken damit auf das System als ganzes." (S. 2)

Diesen strengen Forderungen gemäß konstruiert Bredel ein System, in dem die einzelnen Zeichen klar definierte Funktionen besitzen, sich aber auch voneinander unterscheiden, sodass sie einander stützen und ergänzen. Daraus folgt aber auch nahezu zwangsläufig, dass Bredel Eingriffen in dieses austarierte System mit Argwohn begegnet, denn solche Eingriffe wirken ja „auf das System als ganzes“. Konsequenterweise hält Bredel einige durch die Rechtschreibreform eingeführte Änderungen für problematisch. So knüpft Bredel bei der Beschreibung des Kommagebrauchs an den oben referierten Ansatz von Primus an (S. 68f.), was sie allerdings dazu führt, gewisse durch die Rechtschreibreform eingeführte Liberalisierungen bei der Kommatierung inkohärenter Infinitivkonstruktionen ${ }^{15}$ zu kritisieren, weil diese dazu geführt haben, dass „Satzwertigkeit nicht mehr generell kommaauslösend“ sei (S. 72). Auch hier führt also der Versuch einer linguistischen Analyse direkt zu einer Kritik der geltenden (neuen) Rechtschreibung.

Bredels Vorgehensweise ist ebenso legitim wie die von Primus. Wer sich der Mühe unterzieht, die Systematik von Schreibkonventionen zu ermitteln, wird naturgemäß skeptisch sein gegenüber Bestrebungen, diese Konventionen in einschneidender Weise zu modifizieren. Bredel verhält sich hier nicht anders als ein Biologe, der detailliert das ökologische Gleichgewicht eines Biotops erforscht. Auch dieser wird ja über menschliche Eingriffe in dieses Gleichgewicht wenig erfreut sein.

\section{Abschließende Thesen}

Die in 1.3 formulierten Thesen sollen hier noch einmal bekräftigt und untermauert werden.

1) Eine reine Deskription ohne Norm, d.h. ohne irgendeine Art von Wertung, gibt es nicht. Die Annahme einer ,reinen Beschreibung“ ist ebenso naiv wie die Annahme reiner Empirie ohne Theorie. ${ }^{16}$ Selbst die sich neuerdings zunehmend Gehör verschaffenden ,radikal empirischen' Korpuslinguisten, die fordern, dass a) nur authentische Korpusdaten zulässiges linguistisches Untersuchungsmaterial seien und b) nur

15 Vgl. etwa Deutsche Rechtschreibung (2006: 84f.).

16 Vgl. hierzu ausführlicher Rinas (2011). Das verwickelte Verhältnis von Theorie und Empirie wird in jeder Einführung in die Wissenschaftstheorie diskutiert; vgl. etwa Wuchterl (1987: §1.4) oder Ströker (1992). 
die frequenten sprachlichen Erscheinungen in grammatischen Beschreibungen berücksichtigt werden sollten, ${ }^{17}$ verfahren keineswegs rein induktiv und vorurteilsfrei, denn die Auffassungen a) und b) sind ja ebenfalls Werturteile, die sich keineswegs einfach von selbst verstehen. Man kann es also drehen und wenden, wie man will: Ohne ein Mindestmaß an Norm gibt es keine deskriptive Linguistik.

2) Die Interpunktionspraxis ist ebenso durch (oft unreflektierte) Intuitionen beeinflusst wie andere Formen des Sprachgebrauchs. Auch in den Entwürfen von Interpunktionslehren gab es wiederholt Bemühungen, den faktischen Sprachgebrauch - und somit auch diese Intuitionen - zu erfassen (vgl. Abschnitt 3). Insofern ist auch die Interpunktionslehre nicht einfach willkürlich gesetzt, sondern sie entstand im Rahmen eines langwierigen komplexen Wechselspiels von Beschreibung und Normierung. Somit unterscheidet sich die Entwicklung der Interpunktionslehre qualitativ nicht von anderen Teilbereichen der grammatischen Beschreibung, etwa der Tempus- oder Kasuslehre.

Dieses Oszillieren zwischen Normierung und Beschreibung wird im übrigen auch in der Tradition widergespiegelt, denn es war seit jeher üblich, die Interpunktion sowohl in (tendenziell normativen) Rechtschreiblehren als auch in (tendenziell eher deskriptiven) Grammatiken zu behandeln. Allerdings gab es bei diesen Zuordnungen auch Schwankungen und Unsicherheiten, was man beispielsweise an der diesbezüglich verwirrenden Tradition der von Duden begründeten Werke ablesen kann. Noch in den 1930er Jahren war die Duden-Interpunktionslehre in nahezu identischer Form sowohl im Rechtschreibduden (1934: 38*-45*) als auch in der Duden-Grammatik (1935: 298-314) enthalten. In den nach dem zweiten Weltkrieg erschienen Duden-Grammatiken, d.h. ab der Ausgabe von 1959, ist die Interpunktion hingegen nicht mehr berücksichtigt, sondern vollständig an die Duden-Rechtschreibung delegiert. Jedoch gibt es seit der Duden-Grammatik (2005) wieder Ausführungen zur Interpunktion, wenn auch nur unter dem spezielleren Aspekt der Textkohäsion (S. 1072-1076). Diese Rückbesinnung auf die grammatische Dimension der Interpunktion ist berechtigt; die Interpunktion ist ein Bestandteil der deutschen Grammatik, und zwar der Grammatik der deutschen Schriftsprache.

\section{Literatur}

ADELUNG, Johann Christoph (1782): Umständliches Lehrgebäude der Deutschen Sprache... Bd. 2. Leipzig: Breitkopf.

ADELUNG, Johann Christoph (1788): Vollständige Anweisung zur Deutschen Orthographie... Leipzig: Breitkopf.

ANONYMUS (1754): Über den neuen ,Witz' im Kulturleben (315. Stück). - In: Der Mensch, eine moralische Wochenschrift. Achter Theil. Halle: Gebauer, S. 151-158.

BECKER, Karl Ferdinand (1839): Ausführliche deutsche Grammatik als Kommentar der Schulgrammatik. Dritte Abtheilung. Frankfurt/M.: Kettembeil.

17 Vgl. etwa Kupietz \& Keibel (2009). 
BERGENHOLTZ, Henning (2001): Proskription, oder: So kann man dem Wörterbuchbenutzer bei Textproduktionsschwierigkeiten am ehesten helfen. - In: Andrea Lehr (Hg.): Sprache im Alltag. Beiträge zu neuen Perspektiven in der Linguistik. Herbert Ernst Wiegand zum 65. Geburtstag gewidmet. Berlin: de Gruyter, S. 499-520.

BIELING, Alexander (1880): Das Princip der deutschen Interpunktion nebst einer übersichtlichen Darstellung ihrer Geschichte. Berlin: Weidmann.

BODMER, Johann Jakob (1768): Grundsätze der deutschen Sprache. Zürich: Orell, Geßner und Comp.

BREDEL, Ursula (2011): Interpunktion. Heidelberg: Winter.

BRÜCKER, Jakob (1620): Teutsche Grammatic... Frankfurt: Jennis.

COULMAS, Florian (1981): Über Schrift. Frankfurt/M.: Suhrkamp.

Deutsche Rechtschreibung $(2006)=$ Deutsche Rechtschreibung. Regeln und Wörterverzeichnis. Amtliche Regelung. Hg. v. Rat für deutsche Rechtschreibung. Tübingen: Narr.

DUDEN, Konrad (1876): Versuch einer deutschen Interpunktionslehre zum Schulgebrauch. Beilage zum Jahresbericht des Gymnasiums zu Schleiz. Schleiz: Rosenthal.

Duden-Grammatik (1935) = Der große Duden. Grammatik der deutschen Sprache. Bearb. v. Otto Basler. Leipzig: Bibliographisches Institut.

Duden-Grammatik (1959) = Duden. Grammatik der deutschen Gegenwartssprache. Hg. v. Paul Grebe und der Dudenredaktion. Mannheim: Dudenverlag.

Duden-Grammatik (2005) = Dudenredaktion (Hg.) (2005): Duden. Die Grammatik. 7. Aufl. Mannheim etc.: Dudenverlag.

DÜRSCHEID, Christa (2012): Einführung in die Schriftlinguistik. 4. Aufl. Göttingen: Vandenhoeck \& Ruprecht.

FELBIGER, Johann Ignaz (1774): Anleitung zur deutschen Rechtschreibung. Zum Gebrauche der deutschen Schulen in den kaiserlich=königlichen Staaten. Wien: Deutsche Schulanstalt.

FREYER, Hieronymus (1722): Anweisung zur Teutschen Orthographie. Halle: Waysenhaus.

GÖTZINGER, Max Wilhelm (1839): Die deutsche Sprache und ihre Literatur. Erster Band. Die deutsche Sprache. Zweiter Theil. Stuttgart: Hoffmann.

GRONDIN, Jean (2016) The Hermeneutical Circle. - In: Keane, Niall \& Chris Lawn (Hgg.) The Blackwell Companion to Hermeneutics. Chichester, West Sussex/Malden, MA: Wiley, S. 299305.

HAACKE, Wilmont (1969): Held, Friedrich Wilhelm. - In: Neue Deutsche Biographie. Bd. 8. Berlin: Duncker und Humblot, S. 462f.

HELBIG, Gerhard (1996): Deskription, Regel und Norm in der Grammatikschreibung. - In: Peyer, Ann \& Paul R. Portmann (Hgg.): Norm, Moral und Didaktik - Die Linguistik und ihre Schmuddelkinder. Tübingen: Niemeyer, S. 97-114.

HELD, Friedrich Wilhelm (1844): Aufruf zu einer Revolution der deutschen - Rechtschreibung. Leipzig: Hartknoch.

HENNE, Helmut (1984): Johann Christoph Adelung - Leitbild und Stein des Anstoßes. - In: Bahner, Werner (Hg.): Sprache und Kulturentwicklung im Blickfeld der deutschen Spätaufklärung. Der Beitrag Johann Christoph Adelungs. Berlin: Akademie-Verlag, S. 98-108.

HERGANG, Karl Gottlob et al. (1852): Pädagogische Real-Encyklopädie: oder Encyclopädisches Wörterbuch des Erziehungs- und Unterrichtswesens und seiner Geschichte... Zweiter Band: Haas - Ulrich Zwingli. 2. Aufl. Grimma \& Leipzig: Verlags-Comptoir.

HERLING, Simon Heinrich Adolf (1827): Die Syntax der deutschen Sprache. Zweiter Theil. (Der Periodenbau der deutschen Sprache). 2. Aufl. Frankfurt/M.: Hermannsche Buchhandlung. 
HEYNATZ, Johann Friedrich (1770): Deutsche Sprachlehre zum Gebrauch der Schulen. Berlin: Mylius.

HÖCHLI, Stefan (1981): Zur Geschichte der Interpunktion im Deutschen. Berlin/New York: de Gruyter.

HUDSON, Richard (1984): Invitation to Linguistics. Oxford/Cambridge: Blackwell.

KLEIN, Wolf Peter (2004): Deskriptive statt präskriptiver Sprachwissenschaft!? Über ein sprachtheoretisches Bekenntnis und seine analytische Präzisierung. - In: Zeitschrift für germanistische Linguistik 32/3, S. 376-405.

KUPIETZ, Marc \& Holger KEIBEL (2009): Gebrauchsbasierte Grammatik: Statistische Regelhaftigkeit. - In: Konopka, Marek und Bruno Strecker (Hrsg.) (2009): Deutsche Grammatik - Regeln, Normen, Sprachgebrauch. Berlin/New York: de Gruyter, S. 33-50.

LORBERG, G. A. Ph. (1823): Das Komma: einfache und bestimmte Regeln über den Gebrauch desselben in der deutschen Sprache; nebst einem Anhang über den Unterschied zwischen Komma, Semikolon und Kolon. Frankfurt a.M.: Hermann.

MAITZ, Péter \& Stephan ELSPASS (2007): Warum der ,Zwiebelfisch“ nicht in den Deutschunterricht gehört. - In: Informationen Deutsch als Fremdsprache 34, H. 5/2007, S. 515-526.

MEINUNGER, André (2008): Sick of Sick? Ein Streifzug durch die Sprache als Antwort auf den ,Zwiebelfisch‘. Berlin: Kadmos.

MENTRUP, Wolfgang (1983): Zur Zeichensetzung im Deutschen - Die Regeln und ihre Reform. Oder: Müssen Duden-Regeln so sein, wie sie sind? Tübingen: Narr.

MÖLLER, Johann Georg Peter (1752): Sammlung scherzhafter Versuche. Leipzig: Breitkopf.

MORITZ, Karl Philipp (1796): Allgemeiner deutscher Briefsteller... 2. Aufl. Berlin: Maurer.

PARKES, Malcolm Beckwith (1993): Pause and Effect. An Introduction to the History of Punctuation in the West. Berkeley/Los Angeles: University of California Press.

PRIMUS, Beatrice (1997): Satzbegriffe und Interpunktion. - In: Augst, Gerhard und Karl Blüml (Hrsg.) (1997): Zur Neuregelung der deutschen Orthographie. Begründung und Kritik. Tübingen: Niemeyer, S. 463-488.

QUINTILIANUS: zitiert nach der Ausgabe: Marcus Fabius Quintilianus (2006): Ausbildung des Redners. Zwölf Bücher. Zweiter Teil, Buch VII-XII. Hg. u. übers. v. Helmut Rahn. Darmstadt: Wissenschaftliche Buchgesellschaft.

Rechtschreibduden (1934) = Duden. Rechtschreibung der deutschen Sprache und der Fremdwörter. 11. Aufl. Bearb. v. Otto Basler. Leipzig: Bibliographisches Institut.

Rechtschreibduden (1973) = Dudenredaktion (Hg.) (1973): Duden - Rechtschreibung der deutschen Sprache und der Fremdwörter. 17. Aufl. Mannheim/Wien/Zürich: Dudenverlag.

Rechtschreibduden (1980) = Dudenredaktion (Hg.) (1980): Duden - Die Rechtschreibung. 18. Aufl. Mannheim/Wien/Zürich: Dudenverlag.

Rechtschreibduden (1991) = Dudenredaktion (Hg.) (1991): Duden - Rechtschreibung der deutschen Sprache und der Fremdwörter. 20. Aufl. Mannheim/Leipzig/Wien/Zürich: Dudenverlag.

RINAS, Karsten (2011): Sprache, Stil und starke Sprüche. Bastian Sick und seine Kritiker. Darmstadt: Lambert Schneider/WBG.

RINAS, Karsten (2012): Zur Geschichte der deutschen Interpunktionslehre vom 15. bis zum 17. Jahrhundert. - In: Sprachwissenschaft 37/1, 2012, S. 17-64.

RINAS, Karsten (2014): Von der Rhetorik zur Syntax: Die deutsche Interpunktionslehre im Zeitalter der Aufklärung. - In: Sprachwissenschaft 39/2, 2014, S. 115-181.

RINAS, Karsten (2015): Zur historischen Einordnung von Jakob Brückers Interpunktionslehre. In: Beiträge zur Geschichte der deutschen Sprache und Literatur 137/3, S. 373-395. 
RINAS, Karsten (2016): Die deutsche Interpunktionslehre in der ersten Hälfte des 19. Jahrhunderts. Versuch eines Überblicks. - In: Sprachwissenschaft Bd. 41, Heft 1/2016, S. 31-98.

RINAS, Karsten (i.D.): Gescheiterte Neubegründung und verkappte Kodifizierung: Die deutsche Interpunktionslehre zwischen 1850 und 1915. - Erscheint in: Sprachwissenschaft.

ROSSBERG, Christian Gottlob (1774): Anweisung für die Jugend zur richtigen Aussprache und Rechtschreibung im Deutschen. Dresden \& Warschau: Gröll.

DE SAUSSURE, Ferdinand (1967[1916]): Grundfragen der allgemeinen Sprachwissenschaft. 2. Auflage. Berlin: de Gruyter. [Französische Erstauflage 1916]

STRÖKER, Elisabeth (1992): Einführung in die Wissenschaftstheorie. 4. Aufl. Darmstadt: Wissenschaftliche Buchgesellschaft.

WEISE, Christian (1691): Curiöse Gedancken Von Deutschen Brieffen... Dresden: Mieth.

WEISKE, Johannes (1838): Theorie der Interpunktion aus der Idee des Satzes entwickelt. Leipzig: Reichenbach.

WUCHTERL, Kurt (1987): Methoden der Gegenwartsphilosophie. 2. Aufl. Bern/Stuttgart: Haupt.

doc. Dr. phil. PhDr. Karsten Rinas / K.Rinas@seznam.cz

Univerzita Palackého v Olomouci, Filozofická fakulta, Katedra germanistiky

Kř́žkovského 10, 77180 Olomouc, CZ 